\title{
Decimeter-wave impedance measurement of Y-and Tl-based superconductors with and without an external magnetic field
}

\author{
S E DEM'YANOV, A A DROZD, V R SOBOL AND \\ D V PASHIK \\ Institute of Solid State Physics, P. Brovki 17, Su-220726 Minsk, USSR
}

\begin{abstract}
The real part of low-temperature surface impedance of samples of high- $T_{c}$ superconductors in the $\mathrm{YBa}_{2} \mathrm{Cu}_{3} \mathrm{O}_{6+x}$ ceramic system was studied by the method of registration of the own $Q$-factor of coaxial shortringed cylindrical resonator constructed entirely from examined material. For ceramic samples of high- $T_{c}$ superconductors in the $\mathrm{Tl}_{2} \mathrm{Ba}_{2} \mathrm{Ca}_{2} \mathrm{Cu}_{3} \mathrm{O}$ system the same measurements were made with the help of coaxial $\mathrm{Al}$ resonator. The wavelength of radio frequency field equals $70 \mathrm{~cm}$. The dependence of the impedance of Y-based superconductors on weak magnetic field at helium temperature is close to the square law. The magnitude of impedance at temperature of liquid helium for Y-based superconductors composes $0,04 \mathrm{ohm}$, for Tl-based superconductors $-0,01 \mathrm{ohm}$ respectively which correlates with the statement of the presence of giant resonant absorption of radio frequency field power.
\end{abstract}

Keywords. Ceramic superconductors; surface impedance.

\section{Introduction}

The practical uses of high- $T_{c}$ superconducting materials for transmission devices and storage of radio frequency ( $\mathrm{RF}$ ) field energy have extraordinary importance. The main character of the materials for this purpose is the surface impedance. This is studied under external influence simulating real conditions of exploitation. The present paper reports the influence of temperature and external magnetic field on the impedance of Y- and Tl-based high $T_{c}$ ceramic systems. Similar studies have been done by others (Hagen et al 1987; Fastampa et al 1988; Radlife et al 1988; Fathy et al 1988; Awasthi et al 1988; Tomash et al 1988) in which temperature and magnetic field dependences of impedance $r$ of the above mentioned systems were studied in microwave and higher frequency range.

\section{Experimental procedure}

For our work, the concepts of Radlife et al (1988) were used. However the complexity was in the non-triviality of the resonant system for RF field with wavelength in vacuum of $70 \mathrm{~cm}$ which made it necessary to choose coaxial shortringed resonator, entirely constructed from superconducting material. The Y-based high- $T_{\mathrm{c}}$ superconductor was synthesized according to the reaction:

$$
\mathrm{Y}_{2} \mathrm{O}_{3}+4 \mathrm{BaO}_{2}+6 \mathrm{CuO}=2 \mathrm{YBa}_{2} \mathrm{Cu}_{3} \mathrm{O}_{6+x}+2 \mathrm{O}_{2}
$$

To understand the details of the resonator a technological scheme which included hydrostatic pressing from superconducting powder was chosen. The powder was obtained by choosing the initial components according to the relations cited above. 
After mixing, the powder was placed on a aluminium oxide ceramic boat. The synthesis was carried out in electric furnaces in the presence of air. The product was ground and milled. Further procedure included pressing from superconducting powder, sintering and mechanical work-up.

The technological scheme of synthesis of Tl-based superconducting samples was similar to the synthesis of Y-based samples. The synthesis included two stages. At the first stage the initial compounds $\left(\mathrm{BaO}_{2}, \mathrm{CaO}, \mathrm{CuO}, \mathrm{TlO}_{3}\right)$ were ground and mixed into powder. The powder was then sintered according to the following temperature conditions: smooth heating to $800^{\circ} \mathrm{C}$, keeping at this temperature for 5 hours, followed by cooling to room temperature for $1-2 \mathrm{~h}$ and then ground again. The second stage included heating to $880^{\circ} \mathrm{C}$ for $2-5 \mathrm{~h}$ keeping it for $5 \mathrm{~min}$ at this temperature and cooling to $850^{\circ} \mathrm{C}$ for $5 \mathrm{~h}$ followed by cooling to room temperature for 1-2 h. To measure the Tl-based samples the self $Q$-factor method of coaxial Al resonator was used where the Tl-based sample was placed on shortringed surface i.e. in the maximum of magnetic field of the standing wave. The errors were prevented by changing the inside cavity of the resonator by using an identically similar sample made of coaxial Y-based resonator.

The use of coaxial shortringed resonator as a resonant system made it possible to determine both the dependences of $r$ on temperature $(T)$ and magnetic field $(H)$ and absolute magnitudes of $r$. It is caused by the fact that the theory of that resonator even in terms of the impedance method makes it possible to connect the $Q$-factor of the system with its impedance and form-factor determined by the system's geometry. The form-factor was determined both theoretically and experimentally by measurements of $Q$-factor of the Cu-resonator which was identical in geometry.

\section{Results and discussion}

The dependences of impedance $r$ on $T$ and $H$ are represented in figures 1 and 2. $H$ is shown as a fixed parameter for the curve $r=f(T)$ and $H$ takes the range of values from zero to $70 \mathrm{kOe}$ (figure 1). Magnetic field dependence of $r=f(H)$ is represented at the temperature of liquid helium.

The curve $r(T)$ is characterized by a complicated behaviour even for zero $H$. This is because for $H=0$ the $r(T)$ curve does not display saturation at $T=4 ; 2 \mathrm{~K}$ showing that the losses are not eliminated at low temperatures. This temperature dependence close to linearity at $T / T_{\text {crit }}<0.5\left(T_{\text {crit }}\right.$ is critical temperature) has a definite correlation with $r$ behaviour of $f(T)$ as in Tomash et al (1988) where the real part of impedance has an almost linear dependence on $T$. In strong magnetic field with decrease of temperature at $T<10 \mathrm{~K}$, transition of $r$ to "saturation" is observed and the temperature dependence of $r$ in the region of $T>10 \mathrm{~K}$ increases such that the "slope" of the quasilinear temperature dependence at $10 \mathrm{~K}<T<40 \mathrm{~K}$ becomes larger. According to classical theory the concentration of unpaired electrons increases with increasing $H$ and along with it the appearance of unpaired electrons with temperature obviously "switches on" not immediately, but is the source of the "saturation" of $r(T)$ in the region of $T<10 \mathrm{~K}$ in field of $H=10 \mathrm{kOe}$ and $H=70 \mathrm{kOe}$.

On the field dependence of $r(H)$ (figure 2 ) two characteristic regions were seen: the region of strong increase of $r$ with $H$ and region of weak change of $r$ close to linearity with small angle of slope, extending to maximal magnitude of magnetic field (for 

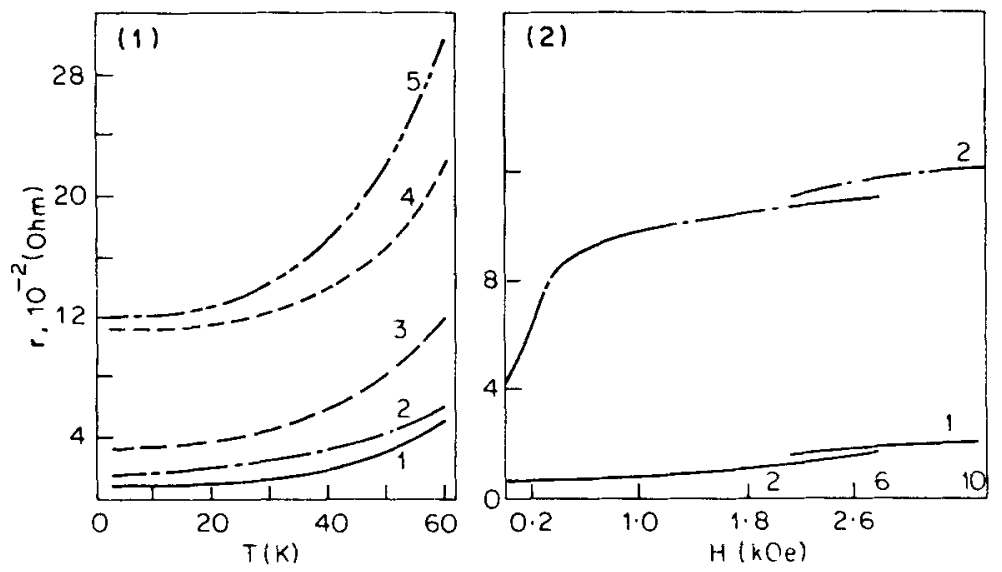

Figures 1 and 2. Influence of temperature $T$ on the surface impedance of $Y$ - and Tl-based superconducting ceramic materials in magnetic field; 1, 2. Tl-based superconductor; 3-5. Y-based superconductor. 1, 3. Magnetic field $\mathrm{H}=0 ; 2,4-\mathrm{H}=10 \mathrm{kOe} ; 5-\mathrm{H}=70 \mathrm{kOe}$. 2. Surface impedance $r$ of $Y$ - and Tl-based superconducting ceramics as a function of external magnetic field at $T=\mathbf{4} \cdot 2 \mathrm{~K} ; 1$. Tl-based and 2 . Y-based superconductor respectively.

$\mathrm{YBa}_{2} \mathrm{Cu}_{3} \mathrm{O}_{6+x}$ samples). The large change of $r(H)$ begins from $H=20 \mathrm{Oe}$ and is close to square law behaviour. This agrees to a certain extent with the results of Heifets et al (1989) on the nature of absorption of RF field energy by similar ceramics in weak magnetic fields as a result of the partial transition of the ceramics from Meissner-like to mixed condition when at $H<H_{\text {crit }}$ the magnetic field on the surface uniformities or unevenness of ceramic resonator is such that their dimensions normal to surface was about $1.5 \times 10^{-3} \mathrm{~mm}$. Starting with the fact that along the surface the dimension of unevenness was of the close value it is quite possible to correlate the appearing absorption with the viscous motion of Abrikosov's vortices. Scherbakov et al (1987) investigated the giant absorption of RF field power in high- $T_{c}$ superconductors. In the normal skin effect approach the magnitude of static conductivity was calculated. The magnitude does not depend on the frequency of the additional absorption mechanism. The "fall" of the static conduction defined like this in the frequency range of $10^{8}-10^{10} \mathrm{~Hz}$ shows the presence of additional absorption mechanism with an effective conductivity $\simeq 10^{3}(\mathrm{ohm} \times \mathrm{cm})^{-1}$ being determined from absorption (frequency of $5 \times 10^{8} \mathrm{~Hz}$ ) at $T=90 \mathrm{~K}$ and $-10^{2}\left(\mathrm{ohm} \cdot \mathrm{cm}\right.$ ) ${ }^{-1}$ at the same frequency at $T=300 \mathrm{~K}$ being the limiting value of Mott insulator. The value of real static conductivity of about $2 \times 10^{3}(\mathrm{ohm} \cdot \mathrm{cm})^{-1}$ at $T=300 \mathrm{~K}$ leads to the magnitude of surface impedance in the free electron approach of about $0.2 \mathrm{ohm}$. The present results also show the presence of peculiarities in absorption not connected with conduction electrons. Therefore, the value of static conductivity $\simeq 10^{4}(\mathrm{ohm} \cdot \mathrm{cm})^{-1}$ at $T=300 \mathrm{~K}$ could cause an $r$ value of about $0.08 \mathrm{ohm}$, while the experiment gives $0.7 \mathrm{ohm}$. This correlates with the results of Scherbakov et al (1987) where the ratio of RF value losses of static conductivity for giant resonant absorption and nonresonant frequency ranges comprises of about $10^{2}$. This is an order of magnitude different from the dependence of surface impedence on static conductivity.

It is worth mentioning that the observed value of the real part of impedance in zero magnetic field of $0.04 \mathrm{ohm}$ at $T=4.2 \mathrm{~K}$ greatly exceeds the impedance reported 
by Awasthi et al (1988) where $r$ measured at a frequency of $10^{10} \mathrm{~Hz}$ corresponds to $0.004 \mathrm{ohm}$. This discrepancy is obviously connected with the fact that samples used by Awasthi et al (1988) were synthesized by the solid-phase reaction:

$$
\mathrm{Y}_{2} \mathrm{O}_{3}+4 \mathrm{BaCO}_{3}+6 \mathrm{CuO}=2 \mathrm{YBa}_{2} \mathrm{Cu}_{3} \mathrm{O}_{6+x}+4 \mathrm{CO}_{2} \text {, }
$$

on this reaction the products have Barium carbonate as an impurity. Moreover their impedance is under doubt, because the value of the specific resistivity at $T=300 \mathrm{~K}$, is $4 \cdot 10^{-3} \mathrm{ohm} \cdot \mathrm{cm}$, a rather great value for this material.

Field dependence $r(H)$ of Tl-based sample differs from that of Y-based sample in that its character does not show any change in region from most weak fields to maximal achievable. In other words there are no sharp changes as it was for Y-based sample in dependence of $r(H)$ on quasi-square rule to linear rule in the range of $H \simeq 600-700$ Oe. The known RF-field absorption mechanisms are viscous motion of vortices (Portis et al 1988), losses on weak bonds between granules and inside them (Khachaturyan K. et al 1987; Dulcic A. et al 1988), motion of hypervortices in the stochastic Josephson medium with strong dependence on external magnetic field in the range of $0-10^{2} \mathrm{Oe}$. This possibly indicates the presence of some new RF field energy absorption mechanism, or some new mechanism of interaction giving a weaker dependence on magnetic field.

\section{Conclusion}

The present results lead us to conclude that the Joule losses i.e. the magnitude of real part of surface impedance $r$ of Y-based and Tl-based ceramic materials at $T=4.2 \mathrm{~K}$ in zero external magnetic field $\left(r^{\mathrm{Y}} \simeq 0.04 \mathrm{ohm}, r^{\mathrm{Tl}} \simeq 0.01 \mathrm{ohm}\right)$ by more than an order excecds the iosses of pure Al with the same class of surface purity (roughness of surface is about $1.5 \times 10^{-3} \mathrm{~mm}$ ), that has $r^{\mathrm{Al}} \simeq 0.002 \mathrm{ohm}$. At room temperature $\mathrm{Y}$-based ceramic resistance composes about $0.7 \mathrm{ohm}$, as compared to $r^{\mathrm{Al}} \simeq 0.01 \mathrm{ohm}$.

The presence of additional nonohm mechanism of losses at temperatures higher than the superconducting transition temperature, that displays discrepancy of static conductivity to surface impedance in the normal skin-effect approach gives us reasons to state that this effect is the cause of the sufficiently low level of selectivity of resonant systems made of given materials at temperatures lower than $T_{\text {crit }}$.

\section{References}

Awasthi A, Carini J, Alavi B and Gruner G 1988 Solid State Commun. 67373

Dulcic A, Crepeau R H and Freed J H 1988 Phys. Rev. B38 8309

Fastampa R et al 1988 Europhys. Lett. 6265

Fathy A, Kalokitis D, Belohoubek E. Sundar H G and Safari A 1988 Phys. Rev. B38 7023

Hagen $\mathrm{M}$ et al 1987 J. Magn. Magn. Mater. $68 \mathrm{Ll}$

Heifets A S. Veinger A I. Zabrodskii A G and Kazakov S V 1989 SU Solid State Phys. 31294

Khachaturyan K et al 1987 Phys. Ret. B36 8309

Portis A M, Blazey K W, Muller K A and Bednorz J G 1988 Europhys. Lett. 5467

Radlife W. Gallop J, Langham C. Gee M and Stewart M 1988 Electron Lett. 241085

Scherbakov A S et al 1987 USSR Fiz. Met. Metalloved. 64742

Tomash W J, Blacstead H A. Ruggiero S T, McGinn P J, Clem J R, Shen K, Weber J W and Boyne D 1988 Phys. Rev. B37 9864 